\title{
Staphylococcus epidermidis pan-genome sequence analysis reveals diversity of skin commensal and hospital infection-associated isolates
}

Sean Conlan ${ }^{1 \dagger}$, Lilia A Mijares ${ }^{1,2,3 \dagger}$, NISC Comparative Sequencing Program ${ }^{4}$, Jesse Becker ${ }^{4}$, Robert W Blakesley ${ }^{4}$, Gerard G Bouffard ${ }^{4}$, Shelise Brooks ${ }^{4}$, Holly Coleman ${ }^{4}$, Jyoti Gupta ${ }^{4}$, Natalie Gurson ${ }^{4}$, Morgan Park ${ }^{4}$, Brian Schmidt ${ }^{4}$, Pamela J Thomas ${ }^{4}$, Michael Otto ${ }^{5}$, Heidi H Kong ${ }^{6}$, Patrick R Murray ${ }^{2}$ and Julia A Segre ${ }^{1 *}$

\begin{abstract}
Background: While Staphylococcus epidermidis is commonly isolated from healthy human skin, it is also the most frequent cause of nosocomial infections on indwelling medical devices. Despite its importance, few genome sequences existed and the most frequent hospital-associated lineage, ST2, had not been fully sequenced.

Results: We cultivated 71 commensal S. epidermidis isolates from 15 skin sites and compared them with 28 nosocomial isolates from venous catheters and blood cultures. We produced 21 commensal and 9 nosocomial draft genomes, and annotated and compared their gene content, phylogenetic relatedness and biochemical functions. The commensal strains had an open pan-genome with $80 \%$ core genes and $20 \%$ variable genes. The variable genome was characterized by an overabundance of transposable elements, transcription factors and transporters. Biochemical diversity, as assayed by antibiotic resistance and in vitro biofilm formation, demonstrated the varied phenotypic consequences of this genomic diversity. The nosocomial isolates exhibited both large-scale rearrangements and single-nucleotide variation. We showed that $S$. epidermidis genomes separate into two phylogenetic groups, one consisting only of commensals. The formate dehydrogenase gene, present only in commensals, is a discriminatory marker between the two groups.

Conclusions: Commensal skin S. epidermidis have an open pan-genome and show considerable diversity between isolates, even when derived from a single individual or body site. For ST2, the most common nosocomial lineage, we detect variation between three independent isolates sequenced. Finally, phylogenetic analyses revealed a previously unrecognized group of S. epidermidis strains characterized by reduced virulence and formate dehydrogenase, which we propose as a clinical molecular marker.
\end{abstract}

\section{Background}

Staphylococcus epidermidis is a common human skin commensal, cultured from virtually every body surface of healthy individuals. The beneficial role of S. epidermidis is demonstrated by its ability to inhibit colonization by the pathogenic Staphylococcus aureus [1]. While S. epidermidis is a commensal on the skin, if it breaches the skin surface and enters the bloodstream, it is considered a pathogen. S. aureus and coagulase negative Staphylococcus, including S. epidermidis, comprise 30\%

\footnotetext{
* Correspondence: Patrick_Murray@bd.com

† Contributed equally

${ }^{1}$ National Human Genome Research Institute, NIH, Bethesda, MD 20892, USA Full list of author information is available at the end of the article
}

of hospital-acquired infections [2], associated with an estimated $\$ 2$ billion annually in treatment costs [3]. S. epidermidis forms biofilms on medical devices, such as contact lenses, catheters, and prosthetic heart valves. The detachment of bacterial cells from biofilms on these devices can lead to bacteremia, with increased morbidity and potential mortality [4]. In clinical settings, Staphylococcal species are frequently resistant to antibiotics, particularly to penicillinase-resistant penicillins (for example, methicillin, oxacillin, nafcillin), constraining treatment options. S. epidermidis is also suspected to be a source of genetic diversity for $S$. aureus to acquire genes enabling better adherence to skin cells [5]. Methicillin-resistant S. aureus (MRSA) is considered a re-emerging pathogen, 
showing increased drug resistance and causing an estimated 18,650 deaths per year in the US [6]. The contrasting roles of $S$. epidermidis in both health and disease make it an important and central player in the human microbiome.

Hospital patients are typically monitored for sepsis from a blood sample. However, commensal staphylococci from the skin can contaminate venipuncture cultures, leading to false positives. This complicates the decision of whether to treat with antibiotics, remove the medical device, or wait-and-see, any of which can extend the patient's hospital stay [4]. One approach to predicting the invasiveness of a strain is the use of marker genes. For instance, the ica operon, contributing to biofilm formation, has been proposed as a marker for invasiveness [7]. The IS256 insertion sequence has also been shown to be associated with biofilm formation and resistance to aminoglycosides [8] and has been proposed as a marker for invasive strains [9]. Despite the statistical significance of these markers, Rohde and colleagues [10] have shown that existing marker genes are not sufficient for discriminating invasive strains in a clinical setting.

While marker-based assays are important tools for epidemiological studies, a deeper understanding of S. epidermidis is required for foundational studies on population structure [10]. Standard microbiological typing methods such as pulsed field gel electrophoresis and multi-locus sequence typing are used to track the spread of strains and measure clonality within patient populations [11], hospitals [12] and geographic areas [13], but are inherently low resolution methods. Microarrays have been used for genome-wide analyses and have successfully identified putative virulence determinants in S. epidermidis strains [14]. New technologies, including optical mapping, which produce ordered restriction enzyme maps, will provide increased physical resolution of strains [15]. Recently, high-throughput sequencing and SNP analysis were used to perform a detailed study of the spread and evolution of MRSA clones [16], demonstrating the power of direct sequencing to study bacterial population dynamics. To date, genome-wide studies on S. epidermidis were hampered by the fact that only two complete reference genomes existed, ATCC12228 [17] and RP62A [18]. Based on the limited number of reference genomes, it was unclear how much sequence diversity existed among S. epidermidis strains.

The pan-genome [19], or collection of genes found among members of a species, is a useful framework for describing genomic diversity within a taxon. For example, Tettelin and colleagues [20] characterized the pan-genome of Streptococcus agalactiae, an important pathogen for newborn infants. Based on the analysis of publicly available genomes as well as genomes generated specifically for their study, they found that approximately $20 \%$ of an individual S. agalactiae genome is made up of genes that are only partially shared with other strains, including unique genes. Furthermore, they found that sequencing additional genomes was predicted to increase the size of the pan-genome, suggesting that $S$. agalactiae has an open genome. This type of analysis has been used to characterize a number of bacterial species [21] as well as genera [22] and has been used to estimate the pan-genome size of all bacteria [23].

To explore the genomic variability of commensal and nosocomial S. epidermidis isolates, we produced draft genome sequences for 30 isolates selected from our extensive strain collection of commensals and pathogens at the NIH Clinical Center. Comparative genomics was used to characterize the pan-genome and deduce the phylogenetic relationship between strains.

\section{Results and discussion}

\section{Genomic variability of commensal S. epidermidis}

As part of our characterization of the human skin microbiome from healthy volunteers (HVs) [24], we cultured $>800$ individual bacterial strains. Based on morphologic characterization, approximately $80 \%$ of those strains were classified as the genus Staphylococcus. We selected $71 \mathrm{~S}$. epidermidis isolates from 15 body sites of seven HVs (Figure 1) to further characterize and identify representative strains for sequencing. The identity of these S. epidermidis strains was confirmed by $16 \mathrm{~S}$ rRNA gene sequencing and ribosomal protein matrix-assisted laser desorption/ionization time of flight (MALDI-TOF)

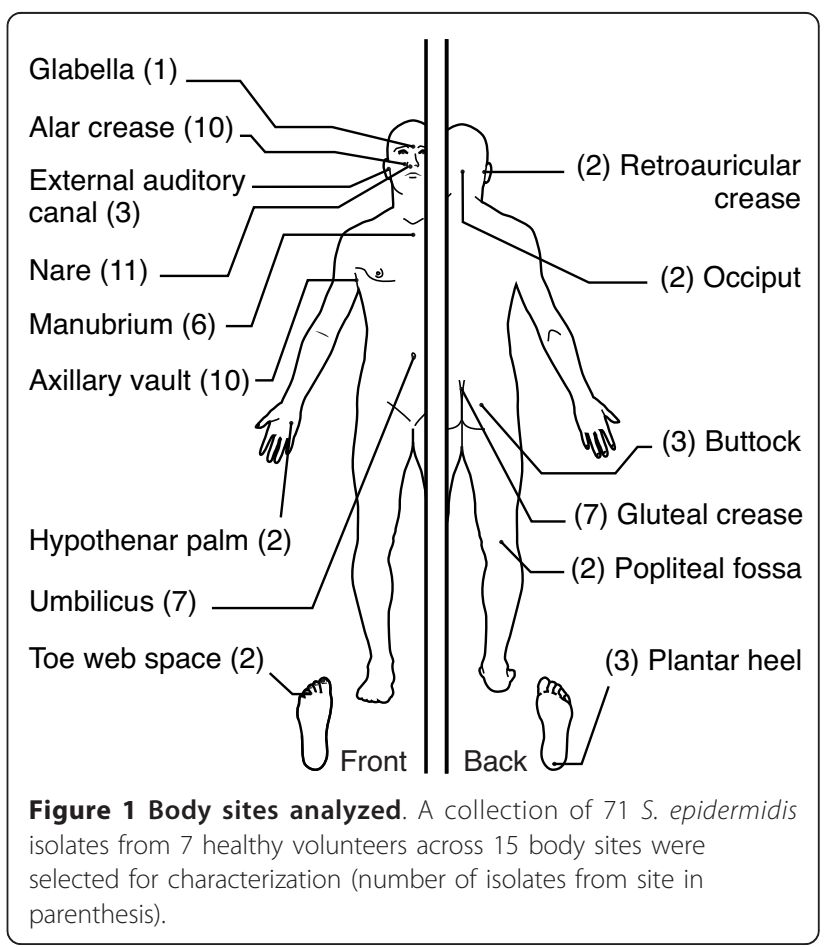


mass spectrometry. In addition, we employed Rep-PCR [25] to investigate the genome architecture of these strains and used these data to select 21 strains for whole genome sequencing (Additional file 1). The $21 \mathrm{draft}$ genomes (including plasmids) were $2.5 \pm 0.1 \mathrm{Mb}$ in size and $32 \%$ GC on average. Each genome had 2,436 \pm 79 predicted protein coding genes. Predicted genes were clustered into orthologous groups, resulting in 3,750 gene clusters and 540 singletons. Gene accumulation curves [20] (Figure 2a) showed that the core genome size fits an exponential decay curve that plateaus at 1,960 genes while the pan-genome data fit a power law curve. The S. epidermidis genome, while relatively constant in size ( $<5 \%$ variance), was $80 \%$ core genes and $20 \%$ variable genes that were drawn from a larger pool, indicating an open pan-genome where each genome sequence added a number of new genes.

The function of the genes within the variable genome was investigated by assigning all gene clusters to clusters of orthologous groups (COG) categories [26]. Three of the 20 COG categories were significantly enriched in the variable genome by Fisher's exact test $(P<0.05)$ : $(1)$ replication, recombination and repair; (2) transcriptional regulators; and (3) defense mechanisms (Figure 2b). Enrichment of these classes was driven by diversity in mobile genetic elements (recombinase and integrase genes), transcriptional regulators, and ABC-type multidrug transporters, respectively. In addition, $40 \%$ of the genes in each genome were not assigned a COG function, reflecting novel gene clusters as well as limitations in COG classification.

\section{Genomic variability of nosocomial S. epidermidis}

To investigate if nosocomial (hospital-acquired) S. epidermidis strains share the same genetic diversity as commensal strains, we sequenced nine nosocomial isolates from the NIH Clinical Center, seven from catheter-associated infections, one from joint fluid and one from lung biopsy tissue (Additional file 2). In clinical epidemiological studies, multi-locus sequence typing is commonly used to classify sub-types based on exact sequence matches at seven housekeeping genes [27]. All nine nosocomial isolates belong to clonal complex 2 (CC2), which comprises $74 \%$ of hospital-acquired infections worldwide and contains the dominant sequence type 2 (ST2) [28]. The isolates in this study include important $\mathrm{CC} 2$ founders and subfounders, as defined by Miragaia and colleagues, including three ST2 strains, two ST5 strains and one ST89 strain. To interrogate the larger S. epidermidis core and pan-genome, gene predictions were generated for these nosocomial isolate genomes, for three draft S. epidermidis genomes generated as part of the Human Microbiome Project (HMP) and for the two complete S. epidermidis reference genomes, yielding a total of 35 genomes (Figure 3).

Phylogenetic relationships between the set of 35 isolates (21 commensal, 9 nosocomial, 3 HMP and 2 reference) were assessed using a maximum-likelihood tree built from 4-fold degenerate codon positions of core genes (Figure 4). The USA300 S. aureus TCH1516 genome was used as the outgroup to root the tree but is not shown in Figure 4. Two of the three ST2 nosocomial isolates sequenced in this study, NIH05001 and NIH05005, were near identical at the nucleotide level for all core genes, but optical mapping showed seven insertions (2 to $40 \mathrm{~kb}$ ) and a number of inversions, translocations and duplications between these strains (Additional file 3). The third ST2 isolate, NIH04008, differed at the nucleotide level (96\% identity across neutrally evolving positions) from the other ST2 isolates and contained a number of insertions, including the SP-beta prophage. Of the six other non-ST2 nosocomial isolates, the lung biopsy isolate NIH051668 was >99\% identical to NIH4008 across neutrally evolving positions of core genes. Three of the catheter-derived isolates (NIH08001, NIH06004, NIH04003) were similarly closely related phylogenetically across core genes. Interestingly, NIHLM020 and NIH05003 shared the same degree of phylogenetic relatedness as above pairs of nosocomial isolates despite coming from commensal and nosocomial isolation sources, respectively. Finally, NIH051475, which was isolated from joint fluid, is the most distantly related of the nosocomial isolates in this study.

\section{Phylogenetic relationship of S. epidermidis commensal and nosocomial genomes}

Strikingly, the 35 S. epidermidis isolates formed two distinct groups, called A and B (Figure 4) with excellent bootstrap support. All of the nosocomial isolates mapped to the upper group A in the phylogenetic tree. In contrast, the commensals were distributed between groups A and $\mathrm{B}$. The 24 commensal isolates fall into 22 different sequence types, including many new sequence types (12/ 22 ), made up of new combinations of alleles as well as novel alleles. When overlayed on the phylogenetic tree, isolates with new sequence types were disproportionately found in the group B (7/8 isolates) compared to group A (7/27 isolates) (Figure 4; $P=0.01$ ). Furthermore, eBurst [29] analysis shows that the majority of group A commensal isolates belong to the $\mathrm{CC} 2$ lineage while none of the sequence types in group $\mathrm{B}$ are connected to the $\mathrm{CC} 2$ lineage.

Only ten genes strictly differentiated group A and B isolates, with nine encoding short proteins of unknown function. The exception was the formate dehydrogenase $(f d h)$ gene, encoding a 983 residue protein, which was found only in the group B isolates. The regions flanking 


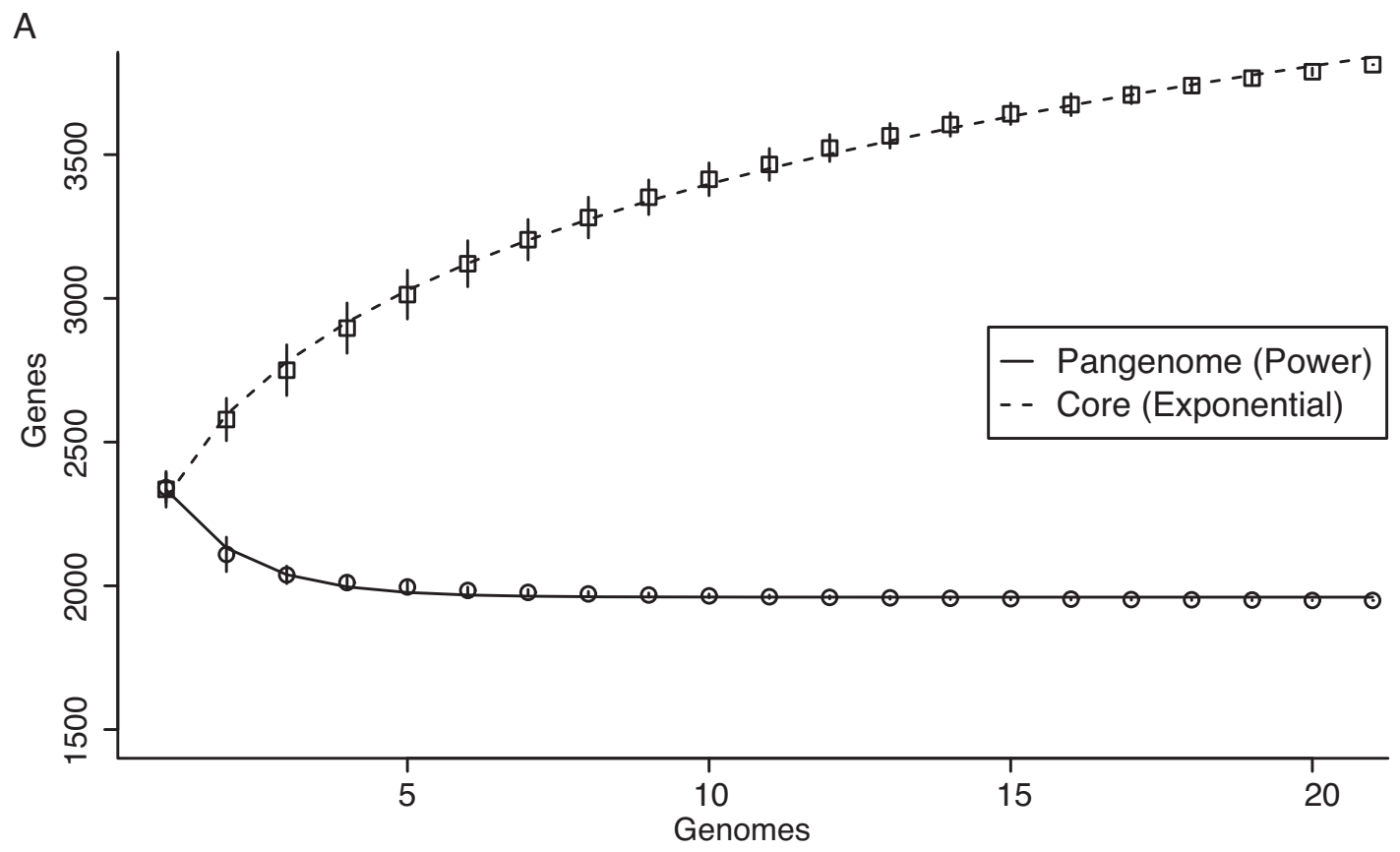

B

* Replication, recombination and repair $(n=231)$

* Defense mechanisms $(n=79)$

* Transcription $(n=203)$

Cell cycle control, cell division, chromosome partitioning $(n=33)$

Function unknown $(n=234)$

Inorganic ion transport and metabolism $(n=235)$

Signal transduction mechanisms $(n=76)$

General function prediction only $(n=417)$

Intracellular trafficking, secretion, and vesicular transport $(n=32)$

Carbohydrate transport and metabolism $(n=194)$

Secondary metabolites biosynthesis, transport and catabolism $(n=55)$

Amino acid transport and metabolism $(n=320)$

Cell wall/membrane/envelope biogenesis $(n=122)$

** Coenzyme transport and metabolism $(n=140)$

Lipid transport and metabolism $(n=81)$

** Energy production and conversion $(n=151)$

** Posttranslational modification, protein turnover, chaperones $(n=85)$

** Translation, ribosomal structure and biogenesis $(n=175)$

** Nucleotide transport and metabolism $(n=85)$

Cell motility $(\mathrm{n}=8)$

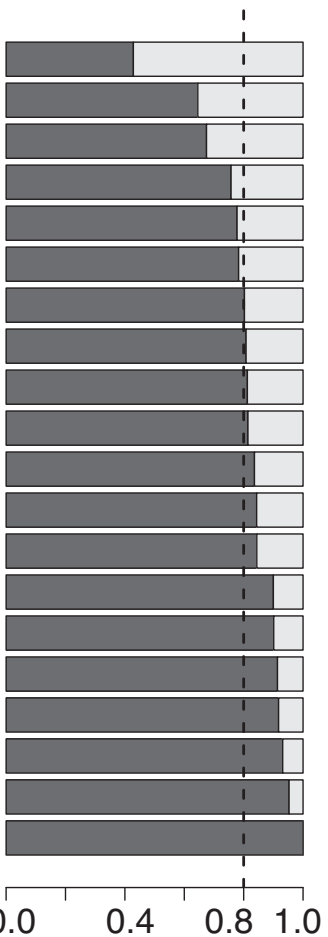

Figure 2 Commensal S. epidermidis has an open pan-genome. Pan-genome size of S. epidermidis. (a) Gene accumulation curves for pangenome (squares) and core-genome (circles) as a function of genomes sequenced $(N)$. Error bars are \pm 1 standard deviation for the 100 simulations. Core gene data are fit by: $y=a \cdot \exp ^{-N / b}+c$. Pan-genome data are fit by: $y=a \cdot N^{b}+c$. (b) Partitioning of core and variable genes for each COG. COGs significantly enriched $(P<0.05$, Fisher exact test) in variable or core genes are marked with single and double asterisks, respectively. The dashed line indicates the expected proportions. 


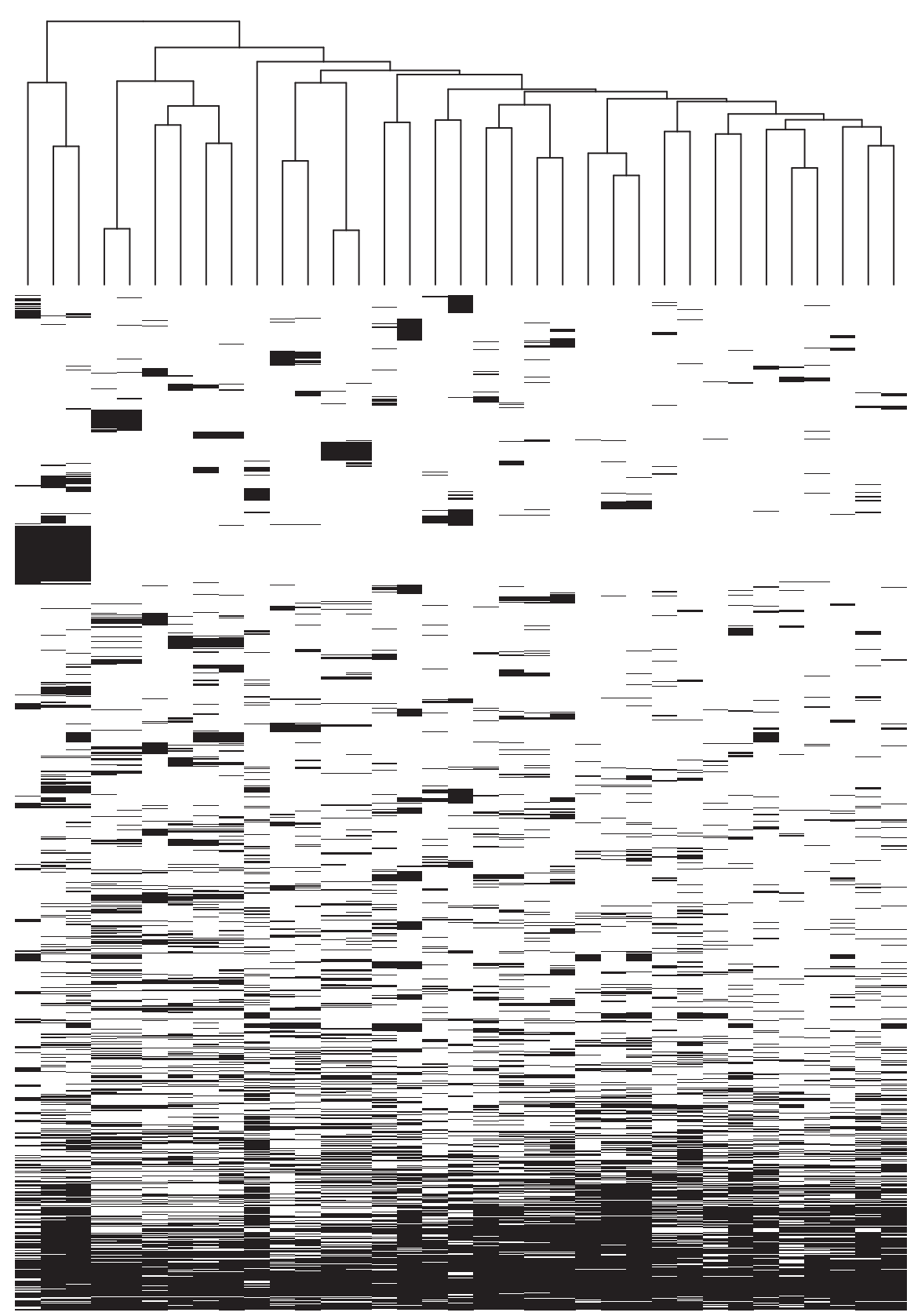

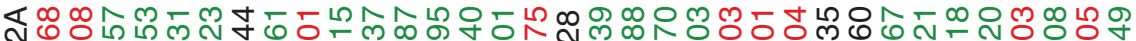

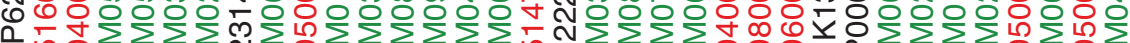

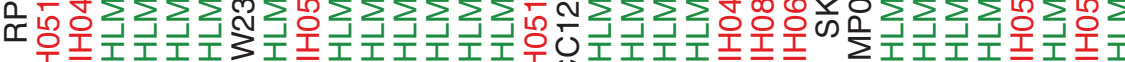

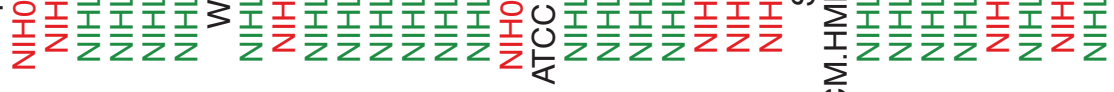

$\sum_{\substack{\infty \\ \hline}}$

Figure 3 S. epidermidis variable genome is dominated by genes shared by few strains. Only the variable genome is shown. Gene clusters present in all genomes or present in only a single genome are omitted. The presence of a gene (and its paralogs) is indicated by a black bar. Nosocomial and commensal isolate names are colored red and green, respectively. The dendrogram was generated using complete linkage clustering of the presence/absence data. 


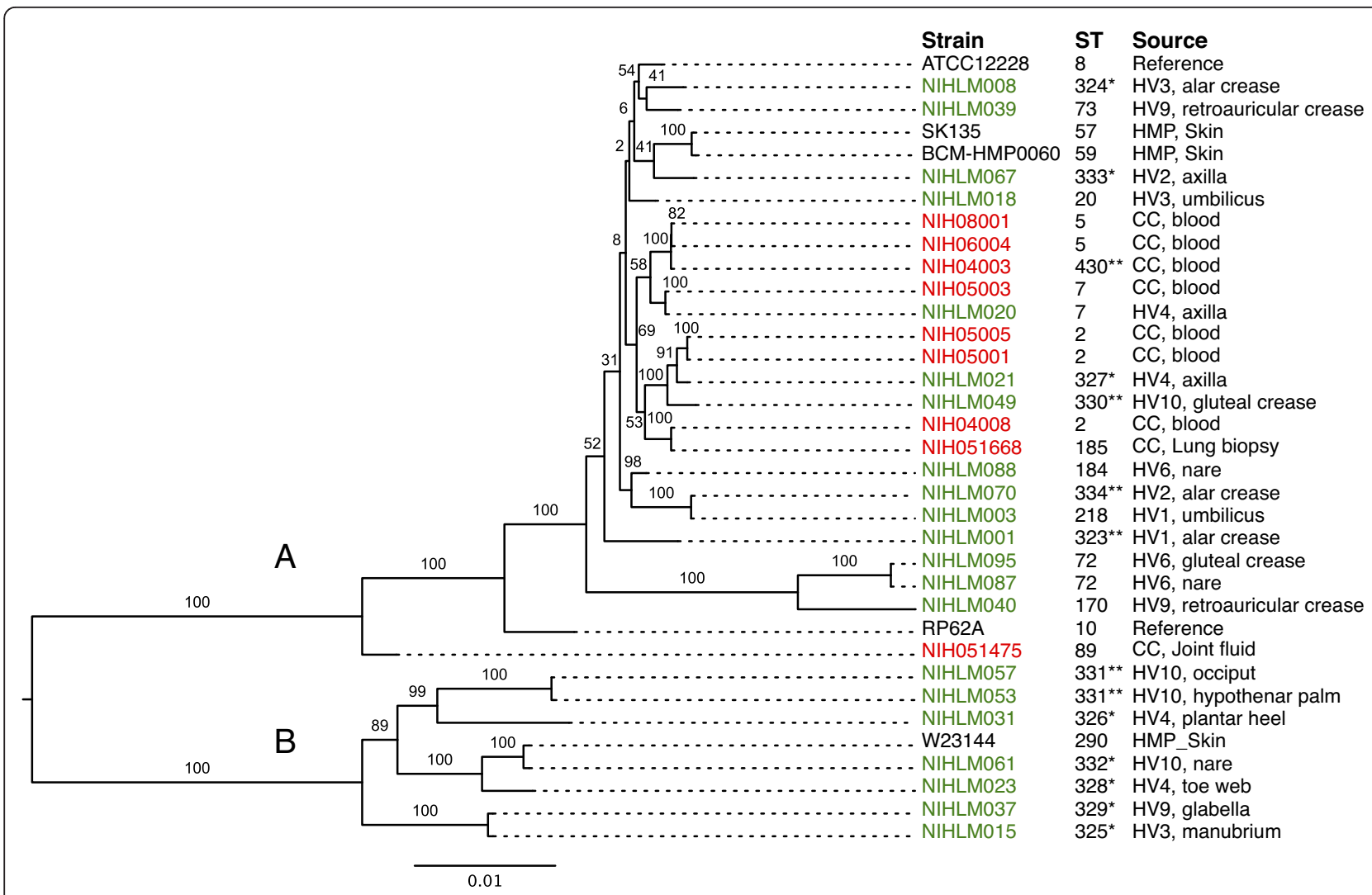

Figure $4 \mathrm{~S}$. epidermidis taxon is made up of two groups. Maximum likelihood phylogenetic tree of S. epidermidis based on four-fold degenerate positions of genes in the core genome. The table shows the sequence type (ST) from multi-locus sequence typing. New sequence types and sequence types with a new allele are indicated by single and double asterisks, respectively. The RP62A and ATCC12228 strains are reference strains (NC_002976, NC_004461). Isolates SK135, BCM-HMP0060 and W23144 were sequenced as part of the Human Microbiome Project Reference Genome catalog. Nosocomial and commensal isolate names are colored red and green, respectively. Bootstrap values are shown on each branch. Outgroup (S. aureus) not shown.

the $f d h$ gene are $98 \%$ identical across all sequenced S. epidermidis isolates and are contiguous in group A isolates (Figure 5). The S. epidermidis fdh gene is $80 \%$ identical to an orthologous $S$. aureus gene with conserved co-linearity, suggesting the $f d h$ gene was lost by group A isolates.

Since the group B isolates are solely commensal in origin, phylogenetically distinct and represent largely novel sequence types, we hypothesized that they could represent an undersampled lineage of $S$. epidermidis, possibly with reduced virulence. We tested this by assaying marker genes in a larger set of 71 commensal strains and 46 nosocomial strains. First, we used PCR to determine the presence of three markers of invasive strains as demonstrated by Rohde et al. [10]: icaA, mecA and IS256. As in Rohde et al., we found these three markers to be significantly associated with nosocomial strains $(P<0.01)$. In slight contrast to Rohde's results, we found icaA, mecA and IS256 at higher rates in our commensal isolates, which reduced their discriminatory power in our isolates (Table 1). Next, we typed our nosocomial and commensal isolates using the $f d h$ gene as a marker and found it in approximately $23 \%$ of commensal strains $(16 / 71)$ but only approximately $4 \%$ of nosocomial strains $(3 / 46)(P=0.02)$. Interestingly, the three $f d h$ positive nosocomial isolates had few virulence markers, as determined by the absence of icaA or mecA genes and two of three with a weak IS256 band. This could suggest that these three isolates, while classified as nosocomials from a blood draw, may actually represent commensal contaminants from the venipuncture. In contrast to traditional nosocomial markers, $f d h$ is a commensal-associated marker with discriminatory potential.

\section{Discriminating commensal and nosocomial isolates}

Group A isolates were a mix of nosocomial and commensal isolates, raising the question of whether differences existed in gene content between the 9 nosocomial and 14 commensal group A strains. While no gene clusters discriminated perfectly, 21 clusters were enriched in genes from nosocomial group A genomes, including nine in the 


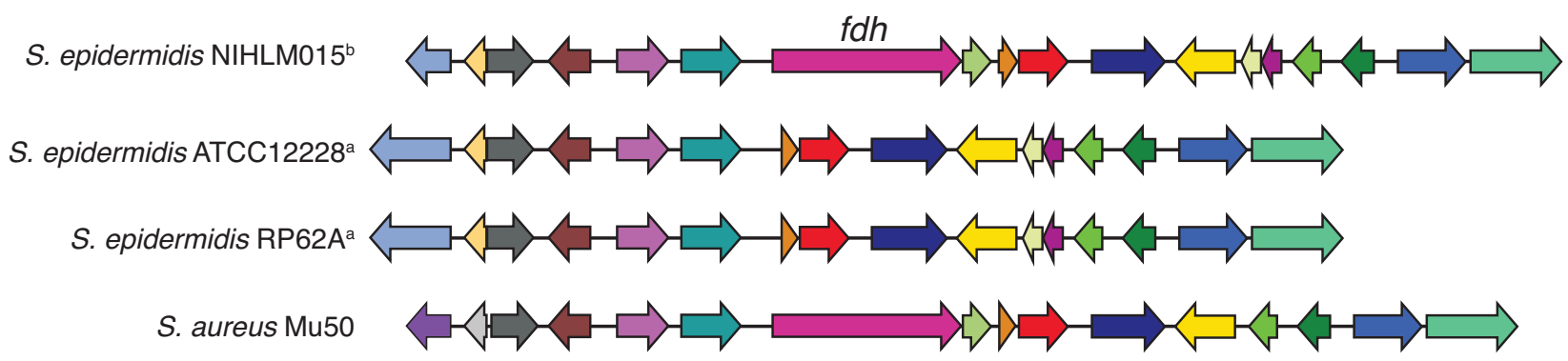

Figure $5 \mathrm{fdh}$ gene neighborhood shows co-linearity across staphylococci. The gene neighborhood of fdh. Strains from group A and B are marked with superscript ' $a$ ' and ' $b$ ', respectively. Genes with conserved function are the same color. Genome alignments were generated using RAST.

SCCmec cassette, the IS256 element and the aacA antibiotic resistance gene. In addition to genes encoding hypothetical proteins, three orthologous gene clusters (RP62A genes SERP0245 to SERP0247) were found in nine nosocomials and only 3 of 14 commensal isolates. These genes encode a putative transport system and transcriptional regulator, possibly associated with antibiotic resistance. As such, while the nosocomial and commensal isolates clustered together phylogenetically, there appeared to be gene content adaptations to hospital life.

We applied two additional analyses to our data to identify patterns differentiating commensal from nosocomial isolates. Principal components analysis was used to visualize the ortholog abundance data shown in Figure 3. In agreement with the phylogenetic tree, group A and B isolates occupied non-overlapping regions of the graph (Figure 6). The group A isolates clustered into an ellipse with isolates forming a gradient from commensal to nosocomial. This clustering suggests that $S$. epidermidis isolates exist on a continuum between benign and pathogenic lifestyles. Patterns in these data were further investigated by using a random forest classifier to separate nosocomial and commensal isolates based on orthologous gene clusters. The random forest was able to classify isolates as commensal or nosocomial with an error rate of $11 \%$. When compared to the principal components analysis data, misclassified isolates were always located in the middle of the cluster where some mixing of commensal and

Table 1 The $f d h$ gene is a marker of commensal strains

\begin{tabular}{|c|c|c|}
\hline & \multicolumn{2}{|c|}{ Prevalence (\%) } \\
\hline & Commensal $(n=71)$ & Nosocomial $^{\mathrm{a}}(n=46)$ \\
\hline Fdh & $22.5 \%(16)$ & $6.5 \%(3)$ \\
\hline$i c a A$ & $33.8 \%(24)$ & $63.0 \%(29)$ \\
\hline mecA & $15.5 \%(11)$ & $80.4 \%(37)$ \\
\hline IS256 & $4.2 \%$ (3) & $47.8 \%(22)$ \\
\hline
\end{tabular}

The prevalence of each gene was surveyed using PCR as described in the Materials and methods. Of 46 nosocomial isolates, 20 were isolated from positive blood culture, 23 from IV catheter and 3 from matched blood and IV catheter. nosocomial isolates is seen. Taken together, these data show that S. epidermidis exists on a continuum of pathogenicity that integrates both phylogeny and gene content.

\section{S. epidermidis biochemical properties: biofilm formation and antibiotic resistance}

S. epidermidis harbors few classical virulence determinants [3], but genes involved in biofilm formation and antibiotic resistance contribute to the persistence of clinical infection [30]. Biofilm formation is assayed in vitro by measuring the bacteria's ability to form a surface-attached aggregation. By this assay, we found that biofilm formation was widespread amongst both commensal and nosocomial $S$. epidermidis isolates. The ica genes, encoding biofilmassociated genes for poly- $N$-acetylglucosamine synthesis, were found in only $60 \%$ of the biofilm-forming commensal isolates, in agreement with previous studies [31]. This suggests genetic heterogeneity underlying the mechanism by which S. epidermidis forms a biofilm. Interestingly, biofilm formation was associated with the site of origin; isolates from moist skin sites demonstrated an enhanced ability to form biofilms compared to those from oily sites (Figure 7). In fact, commensal isolates from moist skin sites have a greater ability to form biofilms in vitro than even those isolated from medical devices.

Antibiotic resistance is common among S. epidermidis isolates and often limits treatment options. Isolates from medical devices were largely resistant to methicillin (21/ 28). Among the commensal isolates examined in this study, methicillin resistance was confined to isolates from HV10 (9/32). Commensal isolates were less likely to be resistant to trimethoprim-sulfamethoxazole (4/32) or clindamycin (2/32) than nosocomial isolates, which were resistant to each drug in $>50 \%$ of isolates tested. First-line antibiotic therapy for catheter-related bloodstream infections is vancomycin. None of the isolates tested were resistant to linezolid, quinupristin-dalfopristin or vancomycin, regardless of isolation source.

A defining feature of methicillin-resistant S. epidermidis (MRSE) is the presence of the SCCmec cassette; a 


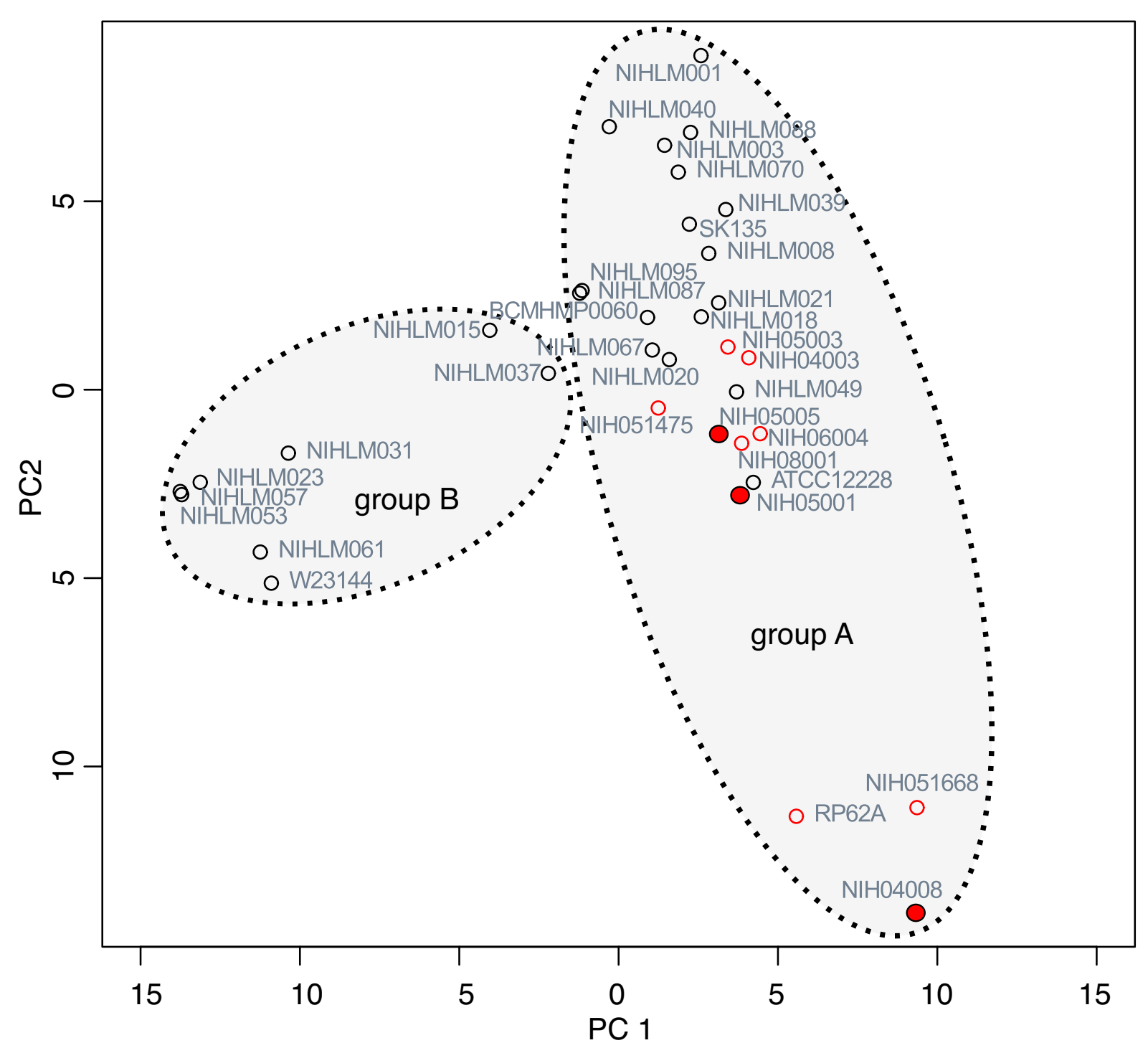

Figure 6 Principal components anaylsis of ortholog clustering. The orthologous gene cluster data for 35 genomes was analyzed using principal components analysis. Nosocomial isolates are shown as red circles and ST2 nosocomial isolates are filled red circles. Commensal isolates are black circles. The dashed ovals indicate the phylogenetic groups described in Figure 4 but are not statistically defined. Percentages of variance explained by PC1 and PC2 are $13.8 \%$ and $9.1 \%$, respectively.

heterogenous sequence defined by combinations of mec gene complexes, cassette recombinases and accessory genes. In agreement with the results of $\mathrm{Li}$ and colleagues [12], the ST2 nosocomial isolates have a type III mec cassette. In fact, all of the nosocomial isolates with an SCCmec cassette (8/9) were either type III or type IV, as found in previous studies of CC2 strains [28]. Both MRSE and methicillin-sensitive $S$. epidermidis (MSSE) isolates were cultured from HV10 across multiple skin sites. Comparing MRSE (9/15) and MSSE (6/15) isolates from HV10, the ability to form biofilms did not correlate with resistance to methicillin, a finding that was not surprising given that biofilm-formation alone confers a degree of antibiotic resistance. Of the sequenced MRSE commensal isolate genomes, NIHLM061 and NIHLM053/057 had type IV and V cassettes, respectively. NIHLM049 had a $m e c A$ gene and partial $m e c R 1$ gene but no detectable cassette recombinase genes. These multiple unique SCCmec cassettes demonstrated the independent acquisition, selection and persistence of multiple MSSE and MRSE isolates within a single individual.

\section{S. epidermidis plasmids and CRISPR}

Mechanisms of resistance to other antibiotics were generally explained by the presence of common resistance genes. A number of these genes are likely carried on 


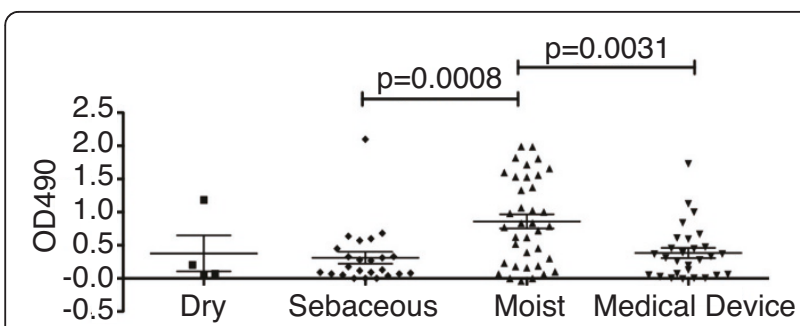

Figure $\mathbf{7}$ in vitro biofilm formation is associated with isolation source. in vitro biofilm formation on polystyrene surface of 71 commensal and 28 medical device (nosocomial) S. epidermidis isolates. Commensal isolates are subdivided by the skin type (dry, sebaceous and moist) as defined in Grice et al. [24]. Each point represents the average of four replicates. Statistical significance was calculated using Mann-Whitney, two-tailed.

plasmids, including pUB110 (bleomycin/kanamycin), pSE12228-01 (tetracycline), and pKH19 (erythromycin). Some resistance phenotypes could not be immediately accounted for by the gene catalog and may be explained by novel genes [32]. For instance, tetracycline resistance is explained by the presence of the pSE12228-01 plasmid in two isolates but three other isolates (NIHLM053/057/061) had no obvious tetracycline resistance genes, suggesting an unidentified gene product may be responsible. For the 30 genomes sequenced in this study, nucleotide homology searches produced strong evidence of 13 different plasmids (free or integrated) in 17 isolates.

In addition, we detected five strains with clustered, regularly interspaced, short, palindromic repeat (CRISPR) loci, which confer sequence-specific immunity to bacteriophage and restrict the spread of conjugative plasmids [33]. CRISPR regions in these five strains contained two to three loci with three to ten spacer regions each. Approximately $90 \%$ of the unique spacers identified in this study did not match targets in the public sequence database, pointing to a large pool of unsequenced staphylococcal phage and plasmids, similar to recently described streptococcal CRISPR diversity [34]. One exception was a nine spacer CRISPR in NIH06004, with five spacers matching plasmid borne proteins, including pre, traE and rep.

\section{Conclusions}

This study presents the largest analysis of S. epidermidis genome sequences to date, and includes both commensal and nosocomial isolates to examine the full pan-genome. Our analysis demonstrates that S. epidermidis has a relatively compact genome with a fixed size of approximately $2.5 \mathrm{Mb}$, and yet as much as $20 \%$ of this genome is in flux, exchanging with a large pool of genes. These values are in agreement with what was reported by Tettelin and colleagues [20] for the S. agalactiae pan-genome and led us to similar conclusions about the importance of sequencing many isolates from a species. Recently, it was shown for Salmonella enterica that core gene variation could be used to construct high-resolution phylogenetic trees, similar to what is shown in Figure 4, that reveal important details not seen by traditional multi-locus sequence typing (MLST) [35]. In addition, they identified a subset of core genes with high sequence variability that appear to be under selection. We also find a small number of core genes with higher than average variability (as measured by average entropy across aligned columns). The etiology of this variability is unclear, but, interestingly, many of the core genes were syntenic gene clusters, including SERP2041-2043 and ureFGD (gene names from the RP62A genome; NC_002976). In a detailed study of the pan-genome of the gut microbe Methanobrevibacter smithii, Hansen and colleagues [36] showed that principal components analysis of orthologous genes was able to cluster strains by family of origin. We did not observe a clustering by individual, but this may be related to the ecological heterogeneity of the skin environment (moist, oily, dry) compared to the gut.

Analysis of the phylogenetic tree based on the 35 genomes in this study showed that S. epidermidis strains clustered into two distinct groups A and B, differentiated by the $f d h$ gene (group B). Group B represents a lineage with reduced virulence, as nosocomial genomes are found only within group A. Moreover, in a larger replication set of 117 S. epidermidis isolates, the $f d h$ gene had discriminatory power as largely absent in nosocomials. While clinical markers have traditionally focused on what 'is' present in nosocomials versus commensals, the $f d h$ gene has the potential to identify possible contaminants in the blood culture from the venipuncture procedure.

Within group A, nosocomial and commensal strains are intermingled phylogenetically, but nosocomials are still enriched in markers traditionally associated with antibiotic resistance. The observation that isolates exist on a gradient of pathogenicity is further supported by principal components analysis of gene content that shows separation based on isolation source (hospital infections versus healthy volunteers). However, strains from both healthy individuals and hospital-associated infections can have nearly identical genomes (for ecample, NIHLM020 from a healthy volunteer's axilla and NIH05003 from a leukemia patient's catheter) indicating that opportunity and environment clearly contribute to hospital-acquired infections. Furthermore, a single individual can carry many S. epidermidis strains with differing antibiotic resistance profiles, capacities to form biofilms and overall gene compositions. These data, combined with the difficulty assigning isolates as strictly commensal or nosocomial, highlights the challenges associated with identifying the genetic determinants of virulence.

The ST2 lineage is the most common hospital-acquired strain. However, it is unclear why this particular lineage 
is dominant. The three ST2 strains sequenced in this study, the first sequences of this dominant sequence type, are similar but not nearly as identical as MRSA USA300 genomes. The only genes we found specifically associated with ST2 strains were part of the type III SCCmec element, which suggested additional roles for this cassette in S. epidermidis virulence. For instance, type III cassettes carry a phenol soluble modulin psm-mec that has been shown to affect the virulence of $S$. aureus [37]. While the acquisition of antibiotic resistance genes and other defense mechanisms may predispose a strain to a pathogenic lifestyle, it may also be a consequence of exposure to a hospital environment.

Metagenomics is one of the ultimate goals of the HMP. While the initial metagenomic sequencing projects relied upon alignment to a limited set of 'reference' genomes, here we clearly show that $S$. epidermidis should be viewed as a pan-genome to empower full metagenomic studies. While complicating a one-to-one assignment of genes to a species, this pan-genomic understanding opens the window to future studies, such as defining the composition of S. epidermidis strains on catheter-derived biofilms and their roles in establishment of infection. This representative catalog of commensal and pathogenic staphylococci from the skin will also empower quantitative assessments of changes in staphylococcal burden during disease exacerbation, such as chronic relapsing atopic dermatitis or MRSA infection. Finally, these results pose the evolutionary question of how and why ST2, the most common nosocomial strain, has been optimized for a pathogenic lifestyle, given the large number of gene combinations available in the S. epidermidis pangenome.

\section{Materials and methods}

\section{S. epidermidis isolates}

Commensal isolates were obtained from swabs of healthy volunteers as described previously [24], classified by $16 \mathrm{~S}$ rRNA gene sequencing and verified with ribosomal protein signature provided by MALDI-TOF mass spectrometry. The DiversiLab Staphylococcus kit for DNA fingerprinting (Bacterial Barcodes Inc., Athens, GA, USA) was used to fingerprint 71 isolates; detection and cluster analysis of fingerprint patterns was performed with DiversiLab software version 3.3. Of 71 isolates, 21 were selected for further characterization based on observed Rep-PCR profiles. Isolates were grown on sheep blood agar and passed three times to confirm homogeneity of the strain. The strains were maintained as frozen glycerol stocks at $-80^{\circ} \mathrm{C}$. Medical device-associated strains were collected between 2004 and 2010 at the NIH Department of Laboratory Medicine. Genomic DNA was prepared using MoBio Laboratories UltraClean Microbial
DNA kit (Mo Bio Laboratories, Inc., Carlsbad, CA, USA) according to manufacturer instructions. DNA was quantified prior to sequencing using the Quant-iT dsDNA BR assay (Invitrogen, Grand Island, NY, USA). All strains sequenced in this study are available from BEI Resources under BEI numbers HM-896 to HM-925.

\section{Functional assays: antibiotic susceptibility and biofilm formation}

MicroScan (Dade Behring, CA, USA) dried MIC/Combo Gram Positive panels were used to evaluate biochemical properties and antibiotic sensitivities based on manufacturer's protocol. Biofilm assays were performed according to Vuong et al. [38]. Briefly, S. epidermidis isolates from a frozen stock were used to inoculate $1 \mathrm{ml}$ of trypticase soy broth, grown overnight and then a 1:100 dilution was prepared in $0.5 \%$ glucose trypticase soy broth. Wells of a microtiter polystyrene plate (BD Falcon, Franklin Lakes, NJ, USA) were inoculated in triplicate. After incubation for $24 \mathrm{~h}$ at $37^{\circ} \mathrm{C}$, the plates were washed twice with double distilled water and stained with $0.1 \%$ safranin for 1 minute. Excess safranin was removed by blotting and air-drying. Absorbance was read at $490 \mathrm{~nm}$ using a SpectraMax 384 (Molecular Devices, Sunnyvale, CA, USA) with SpectraMaxPro software.

\section{Molecular typing}

MLST was carried out using sub-sequences for seven housekeeping genes [27] extracted from the draft genomes. Any allele that did not match an existing allele in the S. epidermidis MLST database [39] was verified by manual examination of the sequence assembly for coverage and quality and Sanger sequencing. New sequence types and alleles were submitted for inclusion in the S. epidermidis MLST database. PCR assays for icaA, $m e c A$ and IS256 were performed as described by Rhode et al. [10]. The $f d h$ gene was detected by PCR by using the following primers: forward, 5'-ATA ATG GTG ATA TTC ATT CG; reverse, 5'-CCG TAT TAG TAA AAG TTC CA. Universal primers against the $16 \mathrm{~S}$ gene were used as a positive control. SCCmec cassette types were determined by comparing all contigs to a database of $\mathrm{SCCmec}$ protein sequences derived from the following GenBank accessions: AB033763, D86934, AB037671, AB063172, AB121219, AF411935, FJ390057. While SCCmec cassettes cannot be definitively subtyped from draft genomes due to fragmentation by multiple transposable elements, a preliminary classification was made based on mec gene complex, ccr gene complex and characteristic joining regions. Types were assigned based on the type descriptions from the International Working Group on the Classification of Staphylococcal Cassette Chromosome Elements (IWG-SCC) [40]. 


\section{Genome sequencing and annotation}

Isolates were sequenced on a Roche 454FLX Ti instrument (Roche Diagnostics GmbH, Mannheim, Germany) by the NIH Intramural Sequencing Center. All isolates were initially sequenced using unidirectional fragment reads; 12 genomes were supplemented with paired-end reads (3 kb insert size). Genomes were assembled using the Roche gsAssembler v2.3 (091027_1459). All genome assemblies exceeded the provisional assembly metrics set forth by the HMP [41]. Specifically, $>90 \%$ of the genome included in the contigs, $>90 \%$ of bases have $>5 \times$ read coverage, $>5 \mathrm{~kb}$ contig N50, $>20 \mathrm{~kb}$ scaffold N50 for genomes with paired ends, $>5 \mathrm{~kb}$ average contig length, $>90 \%$ of core genes found. Optical maps of selected strains were generated by OpGen (Gaithersburg, MD, USA) and used to identify large-scale changes in genome architecture. Glimmer v.3.02 [42] was used to predict protein-coding genes. Orthologous protein clusters were generated using OrthoMCL [43]. Analysis using TBLASTN indicated that relatively few genes were absent due to errors in gene calling or pseudogene formation. Unclustered genes were filtered as described in Lefébure and Stanhope [22]. Briefly, genes were retained as singletons only if they were at least 50 amino acids long and did not have homology to other proteins by BLAST (E-value $<1 e-10)$. Final annotation of each genome was generated using the National Center for Biotechnology Information's (NCBI's) Prokaryotic Genomes Automatic Annotation Pipeline (PGAAP) and further processed by the HMP annotation working group pipeline, which ensures uniform gene product naming. Assemblies and raw sequencing data (SRA) are available from GenBank under BioProject 62343.

COGs [26] were assigned to proteins by first aligning each putative protein sequence against a BLAST database of COG sequences, generated from a file downloaded from the NCBI [44]. A protein was annotated as belonging to a given COG based on the criteria described in Tatusov et al. [26]. Specifically, a protein was assigned to a COG if the best hit in at least two of the COG genomes is annotated to the given COG. CRISPR loci were detected using CRISPRfinder [45] and confirmed by the presence of CRISPR-associated (cas) genes on the same contig. Putative extrachromosomal elements (for example, plasmids) were identified by inspection of paired-end data and BLASTN alignment to the complete plasmid database from NCBI. Antibiotic resistance genes were identified using ARDB [46].

The phylogenetic tree was generated from four-fold degenerate positions in a subset of core gene sequences. Core gene clusters without paralogs were aligned using Muscle v.3.7 [47] and ambiguous alignments were removed. Four-fold degenerate codon positions were extracted from the resulting alignments, filtered to remove positions with low quality bases and concatenated. A phylogenetic tree was built using PhyML v.2.4.4 [48]. Single nucleotide polymorphisms were identified in draft genomes by aligning them to the ATCC12228 genome using ABACAS [49] and then filtering the resultant SNP calls to remove misalignments, homopolymer errors and low quality calls [50].

\section{Statistical tests}

The significance of core gene abundance in COG categories was examined using Fisher's exact test as implemented in $\mathrm{R}$ (v.2.10.0). Principal components analysis was performed using the prcomp function in $\mathrm{R}$. Random forest classification was performed using the randomForest package in R. The P-test [51], as implemented in Unifrac suite was used to determine if environment labels were significantly associated with the structure of the phylogenetic tree of isolates.

\section{Data availability}

This whole-genome shotgun project has been deposited at DDBJ/EMBL/GenBank under BioProject 62343. All genomic sequence data links are individually deposited under the accession numbers: AKGI000000000, AKGJ00000000, AKGK00000000, AKGL00000000, AKGM00000000 AKGN00000000, AKGO00000000, AKGP00000000, AKGQ00000000, AKGR00000000, AKGS00000000, AKGT00000000, AKGU00000000, AKGV00000000, AKGW00000000, AKGX00000000, AKGY00000000, AKGZ00000000, AKHA00000000, AKHB00000000, AKHC00000000, AKHD00000000, AKHE00000000, AKHF00000000, AKHG00000000, AKHH00000000, AKHI00000000, AKHJ00000000, AKHK00000000, AKHL00000000. The version described in this paper is the first version.

Short Read Archive study numbers are: SRP012973, SRP012974, SRP012975, SRP012977, SRP013026, SRP013029, SRP013030, SRP013031, SRP013032, SRP013033, SRP013034, SRP013035, SRP013036, SRP013037, SRP013038, SRP013041, SRP013042, SRP013043, SRP013044, SRP013045, SRP013046, SRP013047, SRP013048, SRP013049, SRP013050, SRP013107, SRP013108, SRP013149, SRP013165, SRP013189.

\section{Additional material}

\footnotetext{
Additional file 1: Genome assembly metrics. The Contig N50 indicates that $50 \%$ of the bases are in contigs of this size or larger. Average coverage is the average number of reads contributing to a given position in the contig.

Additional file 2: Strain metadata. Subject metadata are presented for each strain sequenced in this study. The age column indicates the age of the subject at the time of strain isolation. The subject race/ethnicity is
} 
indicated as white, black or hispanic. For nosocomial isolates, a condensed patient history is provided leading up to strain isolation.

\section{Additional file 3: Optical mapping of ST2 isolates NIH05001 and}

NIH05005. Optical maps were aligned and displayed with the MapSolver 3.1 software. Aligned regions are in blue. Insertions relative to a genome are in white. Inversions and duplications are in red.

\begin{abstract}
Abbreviations
CC2: clonal complex 2; CRISPR: clustered regularly interspaced short palindromic repeats; COG: clusters of orthologous groups; fdh: formate dehydrogenase; HMP: Human Microbiome Project; MALDI-TOF: matrixassisted laser desorption/ionization time of flight; MLST: multi-locus sequence typing; MRSA: methicillin resistant S. aureus; MRSE: methicillinresistant S. epidermidis; MSSE: methicillin-sensitive S. epidermidis; NCBI: National Center for Biotechnology Information; PCR: polymerase chain reaction; SNP: single nucleotide polymorphism; ST: sequence type.
\end{abstract}

\section{Acknowledgements}

We thank the volunteers who participated in this study; C Yang for assistance with sample collection and preparation; E Green, L Marraffini, T Scharschmidt, M Fishback, M Feldgarden and members of the Segre laboratory for valuable comments on the manuscript; The Human Microbiome Project Reference Genome catalog for access to draft sequences for BCM-HMP0060, W23144, SK135. This work was supported by the NHGRI, $\mathrm{NIH} \mathrm{CC}$ and $\mathrm{NCl}$ Intramural Research Programs and $\mathrm{NIH}$ Common Fund Human Microbiome Project grant 1UH2AR057504-01 and 4UH3 AR05750402.

\section{Author details}

'National Human Genome Research Institute, NIH, Bethesda, MD 20892, USA. ${ }^{2}$ Department of Laboratory Medicine, NIH Clinical Center, NIH, Bethesda, MD 20892, USA. ${ }^{3}$ Department of Medical Research and Technology, University of Maryland School of Medicine, Baltimore, MD 21201, USA. ${ }^{4} \mathrm{NIH}$ Intramural Sequencing Center, NIH, Rockville, MD 20852, USA. ${ }^{5}$ Laboratory of Human Bacterial Pathogenesis, National Institute of Allergy and Infectious Diseases, NIH, Bethesda, MD 20892, USA. ${ }^{6}$ Dermatology Branch, Center for Cancer Research, National Cancer Institute, NIH, Bethesda, MD 20892, USA.

\section{Authors' contributions}

SC carried out the genomic analysis studies, and drafted the manuscript. LAM carried out the microbiologic isolation and characterization of the isolates and drafted the manuscript. NISC, JB, RWB, GBB, SB, HC, JG, NG, MP, $B S$, and PJT prepared DNA libraries, performed sequencing and participated in sequence alignment and analysis. MO designed biofilm assays. HHK, PRM and JAS designed and coordinated studies. All authors read and approved the final manuscript.

\section{Competing interests}

The authors declare that they have no competing interests.

Received: 28 March 2012 Revised: 21 July 2012 Accepted: 25 July 2012 Published: 25 July 2012

\section{References}

1. Iwase T, Uehara Y, Shinji H, Tajima A, Seo H, Takada K, Agata T, Mizunoe $Y$ : Staphylococcus epidermidis Esp inhibits Staphylococcus aureus biofilm formation and nasal colonization. Nature 2010, 465:346-349.

2. Hidron Al, Edwards JR, Patel J, Horan TC, Sievert DM, Pollock DA, Fridkin SK: NHSN annual update: antimicrobial-resistant pathogens associated with healthcare-associated infections: annual summary of data reported to the National Healthcare Safety Network at the Centers for Disease Control and Prevention, 2006-2007. Infect Control Hosp Epidemiol 2008, 29:996-101.

3. Otto M: Staphylococcus epidermidis - the "accidental" pathogen. Nat Rev Microbiol 2009, 7:555-567.

4. Wisplinghoff $H$, Bischoff $T$, Tallent SM, Seifert $H$, Wenzel RP, Edmond MB: Nosocomial bloodstream infections in US hospitals: analysis of 24,179 cases from a prospective nationwide surveillance study. Clin Infect Dis 2004, 39:309-317.

5. Diep BA, Gill SR, Chang RF, Phan TH, Chen JH, Davidson MG, Lin F, Lin J, Carleton HA, Mongodin EF, Sensabaugh GF, Perdreau-Remington F: Complete genome sequence of USA300, an epidemic clone of community-acquired meticillin-resistant Staphylococcus aureus. Lancet 2006, 367:731-739

6. Klevens RM, Morrison MA, Nadle J, Petit S, Gershman K, Ray S, Harrison LH, Lynfield R, Dumyati G, Townes JM, Craig AS, Zell ER, Fosheim GE, McDougal LK, Carey RB, Fridkin SK: Invasive methicillin-resistant Staphylococcus aureus infections in the United States. JAMA 2007, 298:1763-1771.

7. Galdbart JO, Allignet J, Tung HS, Rydèn C, El Solh N: Screening for Staphylococcus epidermidis markers discriminating between skin-flora strains and those responsible for infections of joint prostheses. J Infect Dis 2000, 182:351-355.

8. Kozitskaya S, Cho S-H, Dietrich K, Marre R, Naber K, Ziebuhr W: The bacterial insertion sequence element IS 256 occurs preferentially in nosocomial Staphylococcus epidermidis isolates: association with biofilm formation and resistance to aminoglycosides. Infect Immun 2004, 72:1210-1215.

9. Gu J, Li H, Li M, Vuong C, Otto M, Wen Y, Gao Q: Bacterial insertion sequence IS256 as a potential molecular marker to discriminate invasive strains from commensal strains of Staphylococcus epidermidis. J Hosp Infect 2005, 61:342-348.

10. Rohde H, Kalitzky M, Kröger N, Scherpe S, Horstkotte MA, Knobloch JK-M, Zander AR, Mack D: Detection of virulence-associated genes not useful for discriminating between invasive and commensal Staphylococcus epidermidis strains from a bone marrow transplant unit. J Clin Microbiol 2004, 42:5614-5619.

11. Abbassi MS, Bouchami O, Touati A, Achour W, Ben Hassen A: Clonality and occurrence of genes encoding antibiotic resistance and biofilm in methicillin-resistant Staphylococcus epidermidis strains isolated from catheters and bacteremia in neutropenic patients. Curr Microbiol 2008 57:442-448.

12. Li M, Wang X, Gao Q, Lu Y: Molecular characterization of Staphylococcus epidermidis strains isolated from a teaching hospital in Shanghai, China. J Med Microbiol 2009, 58:456-461.

13. Widerström $M$, Monsen $T$, Karlsson $C$, Edebro $H$, Johansson A, Wiström J: Clonality among multidrug-resistant hospital-associated Staphylococcus epidermidis in northern Europe. Scand J Infect Dis 2009, 41:642-649.

14. Yao Y, Sturdevant D, Villaruz A, Xu L, Gao Q, Otto M: Factors characterizing Staphylococcus epidermidis invasiveness determined by comparative genomics. Infect Immun 2005, 73:1856-1860.

15. Latreille P, Norton S, Goldman BS, Henkhaus J, Miller N, Barbazuk B, Bode HB, Darby C, Du Z, Forst S, Gaudriault S, Goodner B, Goodrich-Blair H, Slater S: Optical mapping as a routine tool for bacterial genome sequence finishing. BMC Genomics 2007, 8:321.

16. Harris SR, Feil EJ, Holden MTG, Quail MA, Nickerson EK, Chantratita N, Gardete S, Tavares A, Day N, Lindsay JA, Edgeworth JD, de Lencastre H, Parkhill J, Peacock SJ, Bentley SD: Evolution of MRSA during hospital transmission and intercontinental spread. Science 2010, 327:469-474

17. Zhang $Y-Q$, Ren $S-X, L i ~ H-L$, Wang $Y-X, F u$ G, Yang J, Qin Z-Q, Miao Y-G, Wang W-Y, Chen R-S, Shen Y, Chen Z, Yuan Z-H, Zhao G-P, Qu D, Danchin A, Wen Y-M: Genome-based analysis of virulence genes in a non-biofilm-forming Staphylococcus epidermidis strain (ATCC 12228). Mol Microbiol 2003, 49:1577-1593.

18. Gill SR, Fouts DE, Archer GL, Mongodin EF, Deboy RT, Ravel J, Paulsen IT, Kolonay JF, Brinkac L, Beanan M, Dodson RJ, Daugherty SC, Madupu R, Angiuoli SV, Durkin AS, Haft DH, Vamathevan J, Khouri H, Utterback T, Lee C, Dimitrov G, Jiang L, Qin H, Weidman J, Tran K, Kang K, Hance IR, Nelson KE, Fraser CM: Insights on evolution of virulence and resistance from the complete genome analysis of an early methicillin-resistant Staphylococcus aureus strain and a biofilm-producing methicillinresistant Staphylococcus epidermidis strain. J Bacteriol 2005, 187:2426-2438

19. Bentley S: Sequencing the species pan-genome. Nat Rev Microbiol 2009, 7:258-259.

20. Tettelin H, Masignani V, Cieslewicz MJ, Donati C, Medini D, Ward NL, Angiuoli SV, Crabtree J, Jones AL, Durkin AS, Deboy RT, Davidsen TM, Mora M, Scarselli M, Margarit y Ros I, Peterson JD, Hauser CR, Sundaram JP, 
Nelson WC, Madupu R, Brinkac LM, Dodson RJ, Rosovitz MJ, Sullivan SA, Daugherty SC, Haft DH, Selengut J, Gwinn ML, Zhou L, Zafar N, et al: Genome analysis of multiple pathogenic isolates of Streptococcus agalactiae: implications for the microbial "pan-genome". Proc Natl Acad Sci USA 2005, 102:13950-13955.

21. Tettelin H, Riley D, Cattuto C, Medini D: Comparative genomics: the bacterial pan-genome. Curr Opin Microbiol 2008, 11:472-477.

22. Lefébure T, Stanhope MJ: Evolution of the core and pan-genome of Streptococcus: positive selection, recombination, and genome composition. Genome Biol 2007, 8:R71.

23. Lapierre P, Gogarten JP: Estimating the size of the bacterial pan-genome. Trends Genet 2009, 25:107-110.

24. Grice E, Kong H, Conlan S, Deming C, Davis J, Young A, Nisc Comparative Sequencing Program, Bouffard G, Blakesley R, Murray P, Green E, Turner M, Segre J: Topographical and temporal diversity of the human skin microbiome. Science 2009, 324:1190-1192.

25. Healy M, Huong J, Bittner T, Lising M, Frye S, Raza S, Schrock R, Manry J, Renwick A, Nieto R, Woods C, Versalovic J, Lupski JR: Microbial DNA typing by automated repetitive-sequence-based PCR. J Clin Microbiol 2005, 43:199-207.

26. Tatusov R, Fedorova N, Jackson J, Jacobs A, Kiryutin B, Koonin E, Krylov D, Mazumder R, Mekhedov S, Nikolskaya A, Rao BS, Smirnov S, Sverdlov A, Vasudevan S, Wolf Y, Yin J, Natale D: The COG database: an updated version includes eukaryotes. BMC Bioinformatics 2003, 4:41.

27. Thomas JC, Vargas MR, Miragaia M, Peacock SJ, Archer GL, Enright MC Improved multilocus sequence typing scheme for Staphylococcus epidermidis. J Clin Microbiol 2007, 45:616-619.

28. Miragaia M, Thomas JC, Couto I, Enright MC, de Lencastre H: Inferring a population structure for Staphylococcus epidermidis from multilocus sequence typing data. J Bacteriol 2007, 189:2540-2552.

29. Feil EJ, Li BC, Aanensen DM, Hanage WP, Spratt BG: eBURST: inferring patterns of evolutionary descent among clusters of related bacterial genotypes from multilocus sequence typing data. J Bacterio/ 2004, 186:1518-1530.

30. Schoenfelder SMK, Lange C, Eckart M, Hennig S, Kozytska S, Ziebuhr W: Success through diversity - how Staphylococcus epidermidis establishes as a nosocomial pathogen. Int J Med Microbiol 2010, 300:380-386.

31. Los R, Sawicki R, Juda M, Stankevic M, Rybojad P, Sawicki M, Malm A, Ginalska G: A comparative analysis of phenotypic and genotypic methods for the determination of the biofilm-forming abilities of Staphylococcus epidermidis. FEMS Microbiol Lett 2010, 310:97-103.

32. Sommer MOA, Dantas G, Church GM: Functional characterization of the antibiotic resistance reservoir in the human microflora. Science 2009, 325:1128-1131.

33. Marraffini LA, Sontheimer EJ: Self versus non-self discrimination during CRISPR RNA-directed immunity. Nature 2010, 463:568-571.

34. Pride DT, Sun CL, Salzman J, Rao N, Loomer P, Armitage GC, Banfield JF, Relman DA: Analysis of streptococcal CRISPRs from human saliva reveals substantial sequence diversity within and between subjects over time. Genome Res 2010, 21:126-136.

35. Leekitcharoenphon P, Lukjancenko O, Friis C, Aarestrup FM, Ussery DW: Genomic variation in Salmonella enterica core genes for epidemiological typing. BMC Genomics 2012, 13:88.

36. Hansen EE, Lozupone CA, Rey FE, Wu M, Guruge JL, Narra A, Goodfellow J, Zaneveld JR, McDonald DT, Goodrich JA, Heath AC, Knight R, Gordon J: Pan-genome of the dominant human gut-associated archaeon, Methanobrevibacter smithii, studied in twins. Proc Natl Acad Sci USA 2011, 108(Suppl 1):4599-4606.

37. Queck SY, Khan BA, Wang R, Bach T-HL, Kretschmer D, Chen L, Kreiswirth BN, Peschel A, Deleo FR, Otto M: Mobile genetic elementencoded cytolysin connects virulence to methicillin resistance in MRSA. PLoS Pathog 2009, 5:e1000533.

38. Vuong C, Saenz HL, Götz F, Otto M: Impact of the agr quorum-sensing system on adherence to polystyrene in Staphylococcus aureus. J Infect Dis 2000, 182:1688-1693.

39. Aanensen DM, Spratt BG: The multilocus sequence typing network: mlst. net. Nucleic Acids Res 2005, 33:W728-733.

40. International Working Group on the Classification of Staphylococcal Cassette Chromosome Elements (IWG-SCC): Classification of staphylococcal cassette chromosome mec (SCCmec): guidelines for reporting novel SCCmec elements. Antimicrob Agents Chemother 2009, 53:4961-4967.

41. Provisional Assembly Metrics. [http://www.hmpdacc.org/doc/sops/ reference_genomes/metrics/ProvisionalAssemblyMetrics.pdf].

42. Delcher AL, Harmon D, Kasif S, White O, Salzberg SL: Improved microbial gene identification with GLIMMER. Nucleic Acids Res 1999, 27:4636-4641.

43. Li L, Stoeckert CJ, Roos DS: OrthoMCL: identification of ortholog groups for eukaryotic genomes. Genome Res 2003, 13:2178-2189.

44. COG sequences. [ftp://ftp.ncbi.nih.gov/pub/COG/].

45. Grissa I, Vergnaud G, Pourcel C: CRISPRFinder: a web tool to identify clustered regularly interspaced short palindromic repeats. Nucleic Acids Res 2007, 35:W52-W57.

46. Liu B, Pop M: ARDB - Antibiotic Resistance Genes Database. Nucleic Acids Res 2009, 37:D443-447.

47. Edgar R: MUSCLE: multiple sequence alignment with high accuracy and high throughput. Nucleic Acids Res 2004, 32:1792-1797.

48. Guindon S, Gascuel O: A simple, fast, and accurate algorithm to estimate large phylogenies by maximum likelihood. Syst Biol 2003, 52:696-704.

49. Assefa S, Keane T, Otto T, Newbold C, Berriman M: ABACAS: algorithmbased automatic contiguation of assembled sequences. Bioinformatics 2009, 25:1968-1969.

50. Snitkin ES, Zelazny AM, Montero Cl, Stock F, Mijares L, Murray PR, Segre JA: Genome-wide recombination drives diversification of epidemic strains of Acinetobacter baumannii. Proc Natl Acad Sci USA 2011, 108:13758-13763.

51. Martin AP: Phylogenetic approaches for describing and comparing the diversity of microbial communities. Appl Environ Microbiol 2002, 68:3673-3682.

doi:10.1186/gb-2012-13-7-r64

Cite this article as: Conlan et al: Staphylococcus epidermidis pangenome sequence analysis reveals diversity of skin commensal and hospital infection-associated isolates. Genome Biology 2012 13:R64.

\section{Submit your next manuscript to BioMed Central and take full advantage of:}

- Convenient online submission

- Thorough peer review

- No space constraints or color figure charges

- Immediate publication on acceptance

- Inclusion in PubMed, CAS, Scopus and Google Scholar

- Research which is freely available for redistribution 